\title{
Exploratory factor analysis of a Force Concept Inventory data set
}

\author{
Terry F. Scott* and Daniel Schumayer \\ Department of Physics, University of Otago, Dunedin 9106, New Zealand \\ Andrew R. Gray \\ Department of Preventive and Social Medicine, University of Otago, Dunedin 9106, New Zealand
}

(Received 13 December 2011; published 31 July 2012)

\begin{abstract}
We perform a factor analysis on a "Force Concept Inventory" (FCI) data set collected from 2109 respondents. We address two questions: the appearance of conceptual coherence in student responses to the FCI and some consequences of this factor analysis on the teaching of Newtonian mechanics. We will highlight the apparent conflation of Newton's third law with Newton's first law in one of the FCI questions and suggest an approach to teaching that may avoid this issue. We also note the absence of a distinct factor interpretable as relating specifically to kinematics. Furthermore, we identify and discuss some of the technical difficulties which may be encountered when performing factor analysis on categorical data sets, such as is the case with FCI data sets.
\end{abstract}

DOI: 10.1103/PhysRevSTPER.8.020105

PACS numbers: 01.40.- d, 45.20.D-

\section{INTRODUCTION}

The Force Concept Inventory (FCI) was introduced in 1992 by Hestenes, Wells, and Swackhamer [1] as a successor to and replacement for the Mechanics Diagnostic Test [2]. In 1995 the FCI was updated [3] and has become a generic instrument used worldwide in universities and schools [4]. A large body of research has been based on the use of the FCI [5-7], and a number of teaching methods [8] have been developed using the FCI to determine the effectiveness of the method [9-12].

Early in its history the FCI was subject to criticism as a measure of the conceptual coherence displayed by students [13]. In particular, this criticism focused on whether or not FCI scores could be taken as really reflecting the possession or lack of a Newtonian conception of force. The criticism presented by Huffman and Heller [14] was based on a factor analysis of FCI data collected from a group of 145 high school students and from a group of 750 university students. Their factor analysis, carried out separately for the two groups, indicated that there was no clear factor structure in the data which would justify the claim that students who scored well in the FCI possessed a coherent understanding of the Newtonian concept of force.

The purpose of the present study is twofold. Firstly, it is important to complete the study begun by Huffman and Heller [14], because the presence or absence of conceptual coherence in FCI data is of central importance in measuring students' comprehension. Secondly, an important

\footnotetext{
*scott@physics.otago.ac.nz
}

Published by the American Physical Society under the terms of the Creative Commons Attribution 3.0 License. Further distribution of this work must maintain attribution to the author(s) and the published article's title, journal citation, and DOI. aspect of teaching physics is understanding the difference between the ways that novices and experts think about Newtonian mechanics. It is vital that we, as teachers, understand not only the position from which our students initially approach the mechanical description of the world, but also the effect of our teaching on the way the students think about the world. We hope to show that factor analysis is able to provide valuable information which clarifies these issues.

Factor analysis is a standard technique in the statistical analysis of psychometric and educational data sets and is clearly described in a number of places in the research literature [15-20]. However, it is important to clearly articulate the limitations of this analysis.

Factor analysis provides evidence for the existence of underlying unobserved latent traits. A latent trait is a characteristic or property of the students attempting to answer the FCI questions. The degree to which a student possesses a particular trait determines the likelihood that they will answer a particular question in the FCI correctly. One could find, for example, that a single factor is able to explain most of the variation in the FCI data set. This would be interpreted as meaning that there is a single underlying trait which is polled by the FCI and the score of a student when tested using the FCI is largely determined by the amount of this property that they possess. It should be emphasized that this assignment of meaning to the factors is an interpretive process and that this interpretation may require verification in further investigations.

It is important to distinguish between a factor and a "concept." It is apparent, for example, that the concept of "Newtonian force" is perfectly valid and that the division of this concept into subcategories by Hestenes and Halloun [21] is also perfectly valid on the face of it. The factors found in a FCI data set may or may not agree with 
this division, but this does not imply anything about the conceptual coherence of the FCI itself. The interpretation of FCI questions by experts is just as valid as that by students, and experts agree that the FCI displays the conceptual coherence on which the Newtonian concept of force is built. The important and interesting issue is whether the association of questions seen by an expert agrees with the association of questions seen by students.

In order to prepare the data for factor analysis one faces a significant quantitative problem: the number of necessary respondents required for the statistical methods. It is a standard rule of thumb to use at least 10 times as many respondents as there are variables in the data [22]. The FCI instrument contains 30 questions; therefore, a minimum number of 300 students is needed to produce a reliable result. Our data set meets this requirement by a wide margin (see Sec. II), but given the non-normality of the data this requirement needed to be scaled upwards.

The first step in the exploratory factor analysis is the construction of a correlation matrix between the questions of the FCI. The standard Pearson correlation function may be used, but this correlation function makes the assumption that the data consist of continuous and normally distributed random variables. There is some evidence to suggest that use of the Pearson correlation function on categorical data will significantly underestimate the degree of association between the variables [23]. There are a number of other measures of association, each of which is appropriate to different kinds of data. The polychoric correlation function [24] is a statistical construct which provides a measure of association between categorical variables. When the categorical data are binary, the polychoric correlation function is called the tetrachoric correlation function [24-29].

The tetrachoric correlation function is calculated by assuming that the binary data sample an underlying continuous normally distributed variable. The calculation of the tetrachoric correlation function also assumes that there is some cutoff value of the underlying variable; above this cutoff value the binary variable will register a one, below the cutoff value the binary variable will register a zero. We refer the interested reader to the discussion provided by Schmitt [20]. and for more technical details, see Refs. [30-33].

In the study presented below we use exploratory factor analysis to address two questions: the appearance of conceptual coherence in student responses to the FCI and some consequences of this factor analysis on the teaching of Newtonian mechanics. Section II describes the collection of data, while Sec. III is devoted to the analysis and interpretation of the results.

\section{COLLECTION OF DATA}

The data used in this study were collected from a onesemester physics service course over two years (2008 and 2009). This is a traditional (i.e., passive student), algebra based course designed to provide students entering a variety of health science programs (e.g., medicine, dentistry, pharmacy) with the necessary physics for their professional programs. The FCI was presented to students via the Blackboard online course management system at the end of the mechanics section of the course. The students were not required to complete the survey but were unable to finish their internal assessment for the mechanics component of the course until they had at least viewed the FCI. Collecting data for analysis this way does present some issues as there is no control over the time the students take to complete the survey. Moreover, the students' motivation to answer the questions to the best of their ability is not clear either. However, the course concerned is part of a competitive entry program for a number of professional courses in the health sciences, and the low number of disengaged responses (see following discussion) suggests that the students took the test very seriously.

Prior to the factor analysis the data set was checked and prepared for statistical processing. Responses were evaluated to determine whether or not the student had made a genuine attempt to answer the questions. Each solution set was evaluated on the basis of the number of questions for which no answer was presented, and for which a repeated pattern of answers was detected. The threshold below which students were considered to be disengaged, so that all their records should be removed from the study, was a continuous sequence of 15 missing answers or 15 answers displaying a repeated pattern.

One may be concerned that cleaning the raw data may interfere and influence the outcome of our factor analysis, but we can argue to the contrary. Factor analysis automatically determines the degree of variation in the data which is explained by the postulated set of factors. It is to be expected that disengaged responses will reduce the amount of variation which the analysis is able to explain, but will not affect the validity of the factors which are found in the analysis. Thus one beneficial effect of exploratory factor analysis is that the exclusion of disengaged responses is not critical to the validity of the analysis, so long as a lower value of the percentage of variance in the data explained by the analysis is accepted.

In 2008, 1262 students attended this course, of which 1149 (91\%) completed the FCI. In 2009, 1280 students attended this course, of which 1215 (95\%) completed the FCI. Unfortunately, due to problems with the online learning management system used, only 935 records were accessible from the 2008 data set. The total data set thus contains 2150 records. The overall score ranged from 0 to 30, and the mean being 15.04 (median 14) with a standard deviation of 6.16. Only $41(<2 \%)$ student responses were excluded as being disengaged.

\section{EXPLORATORY FACTOR ANALYSIS}

As a first step in our analysis we determined whether there was a single factor which could explain a significant 
amount of the variation in the data. The next step was to perform a rotation on this correlation matrix to see if there is another set of eigenvectors which are more amenable to interpretation. There are two possibilities in doing the rotation of the initial matrix. We may require that the resulting eigenvectors are orthogonal, so that there is no relationship between the resulting factors. Alternatively, we may allow the rotation to produce a set of possibly nonorthogonal eigenvectors, in which case these factors will be correlated with each other. The second option is in some ways more useful as it provides us with information about the relationship between the factors. As we will see, this may allow us to interpret some factors as having priority over the other factors in some sense.

In an exploratory factor analysis, we let the analysis optimize the number of factors. This may be achieved in several ways. Perhaps the most robust method is "parallel analysis" [34,35] in which we determine the amount of variation explained by the factor set found by the reduction of the correlation matrix. However, there is a point at which a randomly chosen set of matrices will provide as much additional explanation as the factors themselves. Therefore, including further factors cannot actually provide any more explanation of the variation in the data, since a random set of matrices will achieve the same result. Another method is the scree plot technique [36], where the eigenvalues of the correlation matrix are plotted in decreasing order, and one discards those factors which fall in the "scree" of the graph. This method may be represented graphically and nicely illustrates the value of each successive factor in the explanation of the data set (see Fig. 1).

In the paper in which the FCI was first introduced, Hestenes et al. propose a "decomposition" of the concept

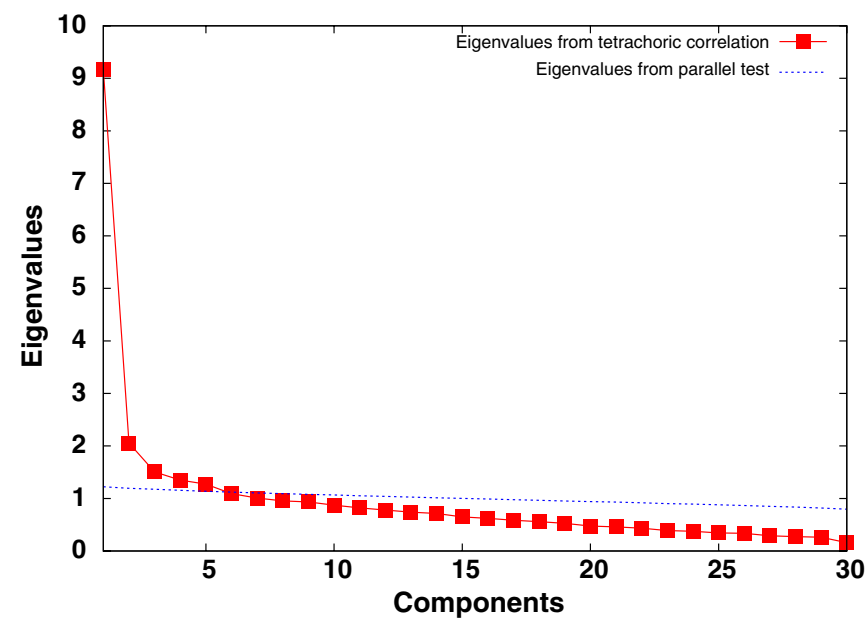

FIG. 1 (color online). The eigenvalues of the tetrachoric correlation matrix are depicted in decreasing order together with the eigenvalues of correlation matrices generated from random uncorrelated standardized normal variables. This figure graphically illustrates the parallel analysis method and suggests that we should look for a five-factor model of the FCI data. of force into "six conceptual dimensions." The questions of the FCI are associated with each of these conceptual dimensions. It is not immediately clear whether the authors of the FCI would anticipate that these conceptual dimensions would be produced by students; these dimensions appear to be a categorization of the FCI questions by the authors based on their understanding of the Newtonian concept of force. Thus it is unclear whether the authors of the FCI would expect to see these conceptual dimensions appearing as factors in a factor analysis of a FCI data set. In a later exchange between Hestenes and Halloun and Heller and Huffman [14,21], it would appear that the authors have no such expectation.

However, this categorization of the FCI questions does provide us with a useful starting point for an investigation into the factor structure of FCI data.

\section{A. Single-factor solution}

The initial factor analysis indicates that a single factor may be taken as underlying student responses. The loading of each FCI question onto this factor is shown in Table I.

The factor loadings indicate the correlation between the question and the underlying factor; it is common practice to accept a correlation of 0.3 or greater as indicating a usable correlation between a particular variable and an underlying factor. From the data presented in Table I it is clear that some questions are more highly associated with an underlying factor than others, questions 13 and 11 being particularly strongly associated with the underlying factor.

We also note that two of the questions have very low loadings onto the underlying factor. These are questions 15 and 26 with loadings of 0.17 and 0.28 , respectively. These loadings are low enough that we would not normally consider that the response to these questions was explained by the existence of an underlying factor. However, these two questions are central to the Newtonian concept of force. Question 15 clearly polls student understanding of Newton's third law and should be associated with an understanding of the Newtonian conception of force. Question

TABLE I. Pattern matrix and unique variances for a one-factor model. Note the low loadings of questions 15 and 26 (underlined).

\begin{tabular}{lccccc}
\hline Question & Loading & Question & Loading & Question & Loading \\
\hline 1 & 0.402 & 11 & 0.707 & 21 & 0.398 \\
2 & 0.327 & 12 & 0.535 & 22 & 0.566 \\
3 & 0.336 & 13 & 0.781 & 23 & 0.580 \\
4 & 0.445 & 14 & 0.516 & 24 & 0.635 \\
5 & 0.596 & 15 & $\underline{0.169}$ & 25 & 0.550 \\
6 & 0.599 & 16 & 0.563 & 26 & $\underline{0.282}$ \\
7 & 0.581 & 17 & 0.538 & 27 & 0.456 \\
8 & 0.478 & 18 & 0.590 & 28 & 0.561 \\
9 & 0.498 & 19 & 0.536 & 29 & 0.337 \\
10 & 0.644 & 20 & 0.596 & 30 & 0.631 \\
\hline \hline
\end{tabular}


26 is more complex in that it investigates several subordinate concepts, namely the linear superposition of forces, the cancellation of forces, and the relationship between acceleration and the net force on an object. In order to understand why these two questions should be so weakly associated with the underlying factor we need to consider the interpretation of this factor.

It is tempting to call this underlying factor "Newtonianness" or something similar. However, all this factor does is measure the tendency for a student to get a question right given that this student has answered another question correctly; i.e., the factor analysis looks for structure in the correlations between correct answers to questions. Thus the lack of association between questions 15 and 26 and the proposed underlying factor should be taken as meaning that answering the other 28 questions correctly does not correlate well with answering questions 15 and 26 correctly. This may mean that while the students have acquired a Newtonian concept of force, they nonetheless tend not to recognize that questions 15 and 26 are related to this concept. However, an alternative interpretation is that these questions are more difficult than the other questions, and that responding correctly to easier questions does not correlate well with responding correctly to more difficult questions. It does in fact appear that question 15 is the most difficult question in the test and this may account for its low loading. Question 26, while it does appear to be quite difficult, is not significantly more difficult than several other questions in the FCI. Question 26 is a relatively complex question simply as a reading exercise, and this may have some effect on students' ability to answer this question correctly irrespective of their possession of a Newtonian conception of force.

\section{B. Rotated factor solution}

We have seen in the previous section that a single underlying factor may be seen as explaining a significant portion of the variation in the response data. We now perform an unconstrained exploratory factor analysis on the data with the goal of determining the optimal number of factors for a multifactor model.

The advantage of such a multifactor model is that it may be easier to interpret than the single-factor model. The optimal number of factors in this model may then be determined using parallel analysis. This analysis suggests that the optimal number of factors to use is five, and this conclusion is clearly supported by the scree plot shown in Fig. 1. The multiple factor model may then be rotated to maximize the loadings of questions onto factors. This rotation may be performed with broadly two kinds of constraint, either the rotation is constrained to preserve the orthogonality of the factors or this constraint is lifted. Here we present two different rotations of the multifactor system, each of which provides us with five factors.

In the first rotation the factors are constrained to be orthogonal, meaning that there is no correlation between the factors. The second rotation presented relaxes this condition and the factors are correlated with each other. In the first case the factor loadings represent the correlation between a question and the factor, in the second case the factor loadings are not so straightforward to interpret as the questions are associated with a given factor which is itself associated with the other factors. However, it seems reasonable to assume that the rotated factors should be correlated with each other as they distinguish aspects of a single underlying factor. For this reason we prima facie expect that the second rotation would more accurately reflect the underlying structure of the data.

In the orthogonal rotation the questions are distributed among factors as shown in Table II (we also show the loading of each question onto its factor).

In the nonorthogonal rotation in which the five factors are correlated, the questions are assigned to factors as shown in Table III.

As we expect that the underlying subconcepts of the overarching Newtonian concept of force will be closely

TABLE II. The assignment of FCI questions to orthogonal factors is shown.

\begin{tabular}{|c|c|c|c|c|c|c|c|c|c|}
\hline \multicolumn{2}{|c|}{ Factor 1} & \multicolumn{2}{|c|}{ Factor 2} & \multicolumn{2}{|c|}{ Factor 3} & \multicolumn{2}{|c|}{ Factor 4} & \multicolumn{2}{|c|}{ Factor 5} \\
\hline Question & Loading & Question & Loading & Question & Loading & Question & Loading & Question & Loading \\
\hline 6 & 0.687 & 5 & 0.621 & 16 & 0.462 & 4 & 0.744 & 19 & 0.436 \\
\hline 7 & 0.708 & 9 & 0.301 & 17 & 0.726 & 15 & 0.678 & 20 & 0.413 \\
\hline 8 & 0.526 & 10 & 0.336 & 25 & 0.673 & 28 & 0.658 & 21 & 0.536 \\
\hline 9 & 0.353 & 11 & 0.551 & & & & & 22 & 0.424 \\
\hline 10 & 0.606 & 13 & 0.741 & & & & & 23 & 0.462 \\
\hline 11 & 0.359 & 18 & 0.738 & & & & & 27 & 0.404 \\
\hline 12 & 0.442 & 22 & 0.362 & & & & & & \\
\hline 16 & 0.508 & 25 & 0.315 & & & & & & \\
\hline 20 & 0.319 & 30 & 0.622 & & & & & & \\
\hline 23 & 0.347 & & & & & & & & \\
\hline 24 & 0.539 & & & & & & & & \\
\hline 29 & 0.382 & & & & & & & & \\
\hline
\end{tabular}


TABLE III. The assignment of FCI questions to nonorthogonal factors is shown.

\begin{tabular}{|c|c|c|c|c|c|c|c|c|c|}
\hline \multicolumn{2}{|c|}{ Factor 1} & \multicolumn{2}{|c|}{ Factor 2} & \multicolumn{2}{|c|}{ Factor 3} & \multicolumn{2}{|c|}{ Factor 4} & \multicolumn{2}{|c|}{ Factor 5} \\
\hline Question & Loading & Question & Loading & Question & Loading & Question & Loading & Question & Loading \\
\hline 5 & 0.654 & 6 & 0.676 & 19 & 0.465 & 16 & 0.442 & 4 & 0.768 \\
\hline 11 & 0.475 & 7 & 0.708 & 20 & 0.428 & 17 & 0.757 & 15 & 0.703 \\
\hline 13 & 0.673 & 8 & 0.510 & 21 & 0.601 & 25 & 0.681 & 28 & 0.651 \\
\hline 18 & 0.803 & 10 & 0.568 & 22 & 0.439 & & & & \\
\hline \multirow[t]{4}{*}{30} & 0.583 & 12 & 0.386 & 23 & 0.493 & & & & \\
\hline & & 16 & 0.451 & 27 & 0.438 & & & & \\
\hline & & 24 & 0.484 & & & & & & \\
\hline & & 29 & 0.378 & & & & & & \\
\hline
\end{tabular}

associated with each other in the data, we will provide an interpretation of the nonorthogonal five-factor model rather than the orthogonal five-factor model. We will also draw some conclusions from the size of the correlations between these factors (shown in Table IV)

We will look at each factor in turn and attempt to interpret them as underlying components of the overall Newtonian force concept. First, we note that six questions, questions $1,2,3,9,14$, and 26, are not assigned to any of these factors. We will treat these questions separately at the end of this section.

Factor analysis does not attempt to account for all of the variation in the data. In this method, variation in a data set is characterized as being due to the effect of one or more underlying factors and some inherent noise. The amount of variation accounted for by the underlying factors is an important result of the analysis, and in our case the second factor, containing questions $6,7,8,10,12,16,24$, and 29 , accounts for about $30 \%$ of the variation in the data. The other factors account for much less, and all factors together account for approximately $40 \%$ of the variation in the data.

\section{Factor 1: Identification of forces}

The questions which are grouped into this factor, questions 5, 11, 13, 18, and 30, are all classified by Hestenes et al. [1] as being about "kinds of force." We prefer to interpret this factor as being concerned with identifying the forces acting on a body. The original classification by Hestenes et al. emphasized the ability of the student to identify the nature of the forces acting on an object, whereas we would suggest that the important characteristic

TABLE IV. Correlation coefficients between factors in the nonorthogonal five-factor model.

\begin{tabular}{lccccc}
\hline \hline & Factor 1 & Factor 2 & Factor 3 & Factor 4 & Factor 5 \\
\hline Factor 1 & 1 & & & & \\
Factor 2 & 0.407 & 1 & & & \\
Factor 3 & 0.509 & 0.342 & 1 & & \\
Factor 4 & 0.445 & 0.357 & 0.348 & 1 & \\
Factor 5 & 0.378 & 0.169 & 0.315 & 0.287 & 1 \\
\hline \hline
\end{tabular}

here is the ability of students to identify all of the forces acting without necessarily employing the extra sophistication of correctly identifying which kind of force is involved. Our contention here is based on the observation that the questions themselves identify the forces for the student and then ask them which of the forces mentioned are in operation. Questions 5, 11, and 18 are classified as asking about solid contact forces, while question 30 involves the idea of air resistance and question 13 is specifically about gravity. We should note that questions 5,18 , and 30 are very similar in form. All of these questions require the student to identify all of the forces acting on a body, and all involve the force of gravity. Question 18 is the question with the highest loading onto the factor and question 11 has the lowest loading.

It would seem from the correlation of the other factors with factor 1 (and the fact that factors 2, 3, 4, and 5 do not correlate as strongly among themselves) that factor 1 has a particular importance in the understanding of the Newtonian conception of force. This is not surprising as the ability to identify forces underlies all other skills involved in the FCI. For example, it is unlikely that a student will be able to determine whether the trajectory followed by an object is straight or curved unless that student is able to identify all the forces acting on that object. The student in this case will also need to understand the effect of a net force on an object as well, but without the identification of forces this concept will not come into play.

\section{Factor 2: Newton's first law with zero force}

Factor 2 may also be interpreted using the schema provided with the FCI. According to this schema questions $6,7,8,10$, and 24 poll student understanding of Newton's first law in the absence of any forces, i.e., that an object will follow a linear path in this case. Question 16 is a question about Newton's third law and loads across two factors, factors 2 and 4 . This question thus has a relatively small loading factor on each of these factors. We will consider this question on its own after our discussion of the five factors in this model. Question 12 relates to kinematics with constant acceleration and question 29 is a "kinds of force" question with the subcategorization of passive solid 
contact forces. These last two questions have lower loadings than the first law questions and we may be tempted to discount them as unimportant. However, a better interpretation of this factor would be that it is a first law factor which takes along with it questions which poll concepts necessary to the application of Newton's first law.

The first law questions in this factor, questions $6,7,8$, 10 , and 24, all relate to curved paths in space. Questions 6, 7 , and 8 rely on the student understanding of those conditions under which an object will describe a linear trajectory. Question 12 is an example of a case where an object will not follow a linear trajectory and is therefore polling the same idea, viz., the distinction between curved and linear trajectories. Questions 10 and 24 ask about the relationship between kinematic concepts and the nature of the object's path; in particular, they test whether the student understands that a straight path corresponds to constant velocity. In this way these two questions may be associated with the distinction between curved and straight paths as they ask about the central distinguishing characteristics of a straight path. The last two questions in this factor, questions 29 and 16, do not appear to fit neatly into this interpretation. However, question 16 loads onto two different factors and should be considered separately in any case. Question 29 has the lowest loading onto factor 2 and we should perhaps expect no more than that there is some aspect of this question that relates to the curved or straight path interpretation rather than a clear unambiguous link. For example, it is clear that an ability to discriminate individual forces is necessary to an understanding of the straight or curved distinction and question 29 requires that the student determine which forces are acting on a stationary object. Questions 6, 7, and 8 require the student to take into account all of the forces acting on an object and to determine that the sum of all of the forces is zero. Hence we can see that in order to determine whether an object will describe a straight or a curved path, the student must be able to sum forces and hence have some understanding of the types of forces involved.

\section{Factor 3: Newton's second law and kinematics}

Factor 3 groups together questions 19, 20, 21, 22, 23, and 27. This is an interesting grouping as it cuts across the classification of Hestenes et al. Questions 19 and 20 are classified as kinematics questions specifically designed to determine whether the student is able to discriminate velocity from position and acceleration. Questions 21 and 22 are classified as questions about Newton's second law; in particular, they are questions in which there is a constant force giving an object a constant acceleration. Question 23 is classified as a first law question in which there is no applied force resulting in a constant velocity. Finally, question 27 is classified as a "kinds of force" question in which students should recognize that friction always opposes motion. It is reasonably clear why questions 19 and 20 are grouped together, as they are very similar questions, and may well be grouped together simply because they ask the student to analyze the same diagram. Similarly, questions 21, 22, and 23 ask the student questions about a single situation and it is possible that the questions are grouped together simply because a student who correctly analyzes the diagram will probably answer all of these questions correctly. However, it is not immediately obvious why these two groups of questions are collected together.

It appears that this factor collects together several groups of questions, each of which poll slightly distinct ideas. Questions 19 and 20 require that the student be able to distinguish velocity from position and acceleration, and is able to represent this distinction graphically. Questions 21, 22 , and 23 do not poll this idea directly, they are quite clearly asking the student about the effect of a continuous force on an object's trajectory, and the effect of removing that force. However, if a student does not have a clear understanding of velocity, acceleration, and position, as well as the ability to represent these ideas graphically, they will not be able to answer these questions - even if they do understand the relationship between force and acceleration. Thus the ability to correctly answer questions 19 and 20 would appear to greatly increase a student's chances of answering questions 21, 22, and 23 correctly. Question 27 would appear to be peripheral to these ideas, being primarily about the nature of friction. However, it does poll the students' understanding of the effect of an unbalanced force on the motion of an object and is thus not entirely out of place in a second law factor.

\section{Factor 4: Newton's first law with canceling forces}

Factor 4 groups together questions 16, 17, and 25. Questions 17 and 25 are categorized as first law questions in which the forces involved cancel so that there is no net force and hence no acceleration. This factor thus looks like a clear first law factor. However, question 16, which is shared with factor 2 , is just as clearly a question about Newton's third law. A careful reading of these questions suggests that an alternative classification should perhaps be considered. In question 16 a truck is being pushed by a car, the car and truck are traveling at constant velocity, and the student is asked about the forces being exerted by the car on the truck and by the truck on the car. This is clearly a situation in which the third law is required for a complete analysis. In question 17 an elevator is pulled up an elevator shaft by a cable at constant velocity. The student is asked about relative sizes of the upward force exerted by the cable and the downward gravitational force. Both of these forces are exerted on the elevator, but they are not an action-reaction pair, and hence the third law does not apply. Similarly, in question 25 a woman pushes a large box along a horizontal floor with constant velocity and the student is asked about the relative sizes of the force exerted 
by the woman and the other forces which are exerted on the box. This is clearly a question about Newton's first law as none of the forces mentioned in the question are members of action-reaction pairs. The commonality between questions 16, 17, and 25 is the cancellation of component forces resulting in a zero net force and a resultant constant velocity. If question 16 did not appear in this factor we would feel completely justified in calling this a first law with canceling forces factor. We feel that this is still an appropriate designation and will explain the inclusion of question 16 in this factor in a separate discussion.

\section{Factor 5: Newton's third law}

Finally, we consider the last factor, factor 5 . This factor is the easiest to interpret and consists of questions 4,15 , and 28. These questions along with question 16 are designed to poll student understanding of Newton's third law. It is worth noting that each of the questions included in this factor poll the third law in situations in which the acceleration of the bodies involved is not zero. Question 16 by contrast requires an understanding of Newton's third law in a situation where all forces cancel and there is no net acceleration.

\section{Conflation of Newton's first and third laws}

Question 16 appears in both factor 2 and factor 4 . These two factors both contain a number of questions relating to Newton's first law. Our factor analysis thus indicates that students who provide the correct answer to questions about Newton's first law are also likely to answer question 16 correctly. However, there is no such relationship between the ability to answer the other questions about Newton's third law and the ability to answer question 16 correctly. To put this point slightly differently, students who answer question 16 correctly treat it as a question about Newton's first law, not a question about Newton's third law. We will here propose an hypothesis to explain this conflation, but we emphasize that this hypothesis requires further investigation.

As "experts" in Newtonian mechanics it will be clear to physics teachers that Newton's third law is in operation in question 16. It is thus natural to assume that students must recognize that the force exerted by the car on the truck is equal and opposite to the force exerted by the truck on the car if they are to answer this question correctly. However, it would appear from our factor analysis that students are able to answer this question correctly for the wrong reason. We would suggest that students follow a chain of reasoning which employs Newton's first law rather than Newton's third law and which is thus incorrect, but which consistently supplies the correct answer. This chain of reasoning is most likely something like the following:

The car and the truck are travelling at constant velocity, therefore the net force on the car and the net force on the truck are zero. The net force on the car is due to the force exerted on it by the truck and vice versa, hence the force exerted on the truck by the car and the force exerted by the car on the truck must be equal and opposite.

In other words, we would suggest that students derive the correct answer, using Newton's first law, that Newton's third law would give them directly. It is interesting that this (faulty) derivation, if applied to question 15, which relates to the same situation, would give the wrong answer. Thus answering question 16 correctly using this reasoning would not improve a student's ability to answer question 15 correctly. Similarly the ability to answer question 15 correctly would not help a student answer question 16 correctly. This would go some way to explaining why questions 16 and 15 are not grouped in the same factor.

\section{Kinematics}

It is interesting to note that the questions listed by the authors as being kinematics questions, questions 9, 12, 14, $19,20,21$, and 22, do not form a distinct factor. Clearly there are a number of questions in the FCI which do not cleanly poll a single concept. It is not possible to ask a question about the second law which does not also require that the student understand the basic kinematics concepts. This applies to most of the kinematics questions as much as to the dynamics questions. However, questions 19 and 20 are purely kinematic and require no understanding of any concepts apart from the kinematic ideas of position, velocity, and acceleration. These questions are not linked to each other in a factor; they are quite strongly linked to factor 4 , the second law factor. Two other kinematics questions (21 and 22) are linked to this factor; these are questions which are related to curved paths. Two of the remaining kinematics questions (questions 9 and 14) do not load onto any factor and the other (question 12) loads onto factor 2, the first law with zero force factor.

Thus it would appear that students associate kinematics ideas much more strongly with the dynamical ideas that use them than to each other.

\section{E. Unassigned questions}

In the unrotated, single-factor model of the FCI data presented here, two questions have low loadings onto the underlying factor. These are questions 15 and 26. Question 26 has a loading of 0.28 and so only just falls below our criterion of 0.3 , below which we consider a question to not be represented by the factor. We will still look carefully at question 26 as it is also absent in the rotated factor model. Question 15 polls an understanding of Newton's third law and question 26 polls Newton's second law.

To understand the very low loading of question $15(0.169)$ it is worth noting that this is hardest question in the FCI in the sense that only $21 \%$ of students answered this question correctly [6]. Figure 2 illustrates the difficulty 


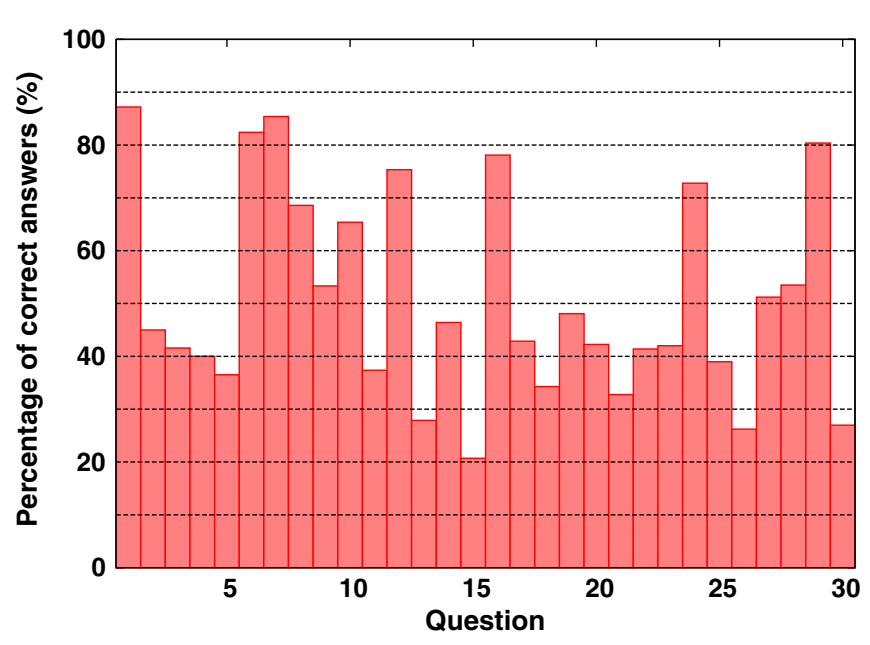

FIG. 2 (color online). Ratio of the correct answers depicted in percentage of the total number of respondents. Horizontal axis represents the questions.

of the FCI questions using the proportion of students who answer a question correctly as a rough measure of difficulty. Furthermore there is a distinct gap in difficulty between question 15 and the next hardest questions, the next hardest question being answered correctly by $25 \%$ of students. Thus the exclusion of question 15 from the single-factor solution could simply be due to the fact that answering any of the other 29 questions in the FCI correctly does not correlate with a correct response to questions 15 simply because this question is so much harder than the other 29. In general, students find questions requiring an understanding of Newton's third law much more difficult than questions involving the first or second laws, or kinematics. Furthermore, this question also involves a pair of objects which are accelerating, this further increases the difficulty of the question for students.

In the five-factor model several questions which are present in the single-factor model do not load onto any factors. These are questions 1, 2, 3, 9, 14. Question 26 is not loaded onto any factors in either the five-factor model or the single-factor model. We will consider this question separately.

The questions which are unassigned in the five-factor model, apart from question 26, clearly poll an underlying Newtonian concept as they appear in the single-factor model. Their disappearance in the five-factor model merely indicates that students do not assign them to any particular substructure of this overarching concept. Questions 1, 2, and 3 are all categorized as polling gravity as a Newtonian force. They could thus be seen as applications of ideas which are more precisely polled in other questions. Questions 9 is about Newton's second law with an impulsive force but it is quite difficult to interpret what this question is asking. We would suggest that this question falls out of the rotated factor model due to the fact that it polls an extraneous factor, the level of reading sophistication of the student. Question 14 is classed as a question about kinematics, specifically polling the understanding that constant acceleration results in a parabolic orbit. This question would appear to the expert as being very similar to question 12 , and in the unrotated model these two questions have very similar loadings. However, it would seem that students do not see questions 12 and 14 as being particularly similar. Furthermore, the exclusion of question 14 from the five-factor model may well be an artifact of the numerical method used to perform the factor rotation. We note that alternative rotation methods do not exclude question 14 from the five-factor model but place this question in factor 3 , the second law factor, rather than into factor 2 with question 12 .

It is not clear why question 26 is excluded from both the single-factor and the five-factor models. Any suggestions that we make here should be taken primarily as suggestions for further research. Question 26 involves a system in which an extra external force is added to an object traveling at constant velocity. It may be that there is some alternative approach to this problem which provides a correct solution using incorrect reasoning. This would lead to a situation where the correct solution to this question would be correlated to ideas outside the collection of concepts involved in the Newtonian conception of force. The only viable way to check this conclusion would be a detailed qualitative study of student responses to questions of this form. Finally, we note that question 26 shares some of the linguistic features of question 9, and could possibly require a higher level of reading sophistication than the other questions in the FCI. Thus, this question may not load onto any of the factors in our analysis as the ability to answer it correctly correlates with an underlying trait which is not involved in the other questions to the same degree, viz., reading sophistication.

\section{CONCLUSIONS}

This analysis broadens our understanding of FCI data and also suggests some interesting features in the conceptual understanding of Newtonian mechanics among novices. Firstly, FCI data show conceptual coherence which manifests in the appearance of stable, interpretable factors. Our analysis clearly confirms that FCI response data may be modeled using a single-factor model, and also supports the use of a five-factor model. In both models, the factor structure explains about $40 \%$ of the variation in the data set. The level of variation explained by the underlying factor structure should perhaps be taken as a minimum. The data for this study were collected in such a way that students had only weak incentives to answer the questions correctly. We have attempted to remove disengaged responses as discussed above; however, pseudorandom response patterns cannot be detected in this way and would contribute significantly to the background noise in the data set. 
Our factor analysis indicates the presence of significant correlations among the factors. However, the factor structure found in our analysis is suggestive rather than conclusive. For this reason most of our conclusions take the form of suggestions for further experiments or for approaches to teaching Newton's concept of force which may be useful.

It appears that Newton's third law is closely (but incorrectly) associated with Newton's first law. This suggests that great pains should be taken to distinguish problems requiring Newton's third law from problems which require Newton's first law. This issue is specifically highlighted in problems in which Newton's third law is applied to constant velocity situations.
Finally, it also appears that the ideas of kinematics are not well differentiated by students from Newton's first and second laws. In particular, students seem to struggle to clearly identify kinematic ideas in the areas of dynamics in which the former concepts are used. This suggests that kinematic concepts should be clearly distinguished from dynamical concepts during the introduction of Newton's first and second laws.

\section{ACKNOWLEDGMENTS}

The authors are indebted to Paul Yates for fruitful and enlightening discussions. This work was funded by the University of Otago.
[1] D. Hestenes, M. Wells, and G. Swackhamer, Force concept inventory, Phys. Teach. 30, 141 (1992).

[2] I. A. Halloun and D. Hestenes, The initial knowledge state of college physics students, Am. J. Phys. 53, 1043 (1985).

[3] Available from http://modeling.asu.edu/R\&E/Research .html.

[4] The latest version of FCI is available at modeling.asu.edu/ R\&E/Research.html.

[5] R. R. Hake, Interactive-engagement versus traditional methods: A six-thousand-student survey of mechanics test data for introductory physics courses, Am. J. Phys. 66, 64 (1998).

[6] M. Planinic, L. Ivanjek, and A. Susac, Rasch model based analysis of the Force Concept Inventory, Phys. Rev. ST Phys. Educ. Res. 6, 010103 (2010).

[7] J. Wang and L. Bao, Analyzing force concept inventory with item response theory, Am. J. Phys. 78, 1064 (2010).

[8] V. P. Coletta, J. A. Phillips, and J. J. Steinert, Interpreting force concept inventory scores: Normalized gain and SAT scores, Phys. Rev. ST Phys. Educ. Res. 3, 010106 (2007).

[9] A. Savinainen and J. Viiri, The Force Concept Inventory as a measure of students conceptual coherence, Int. J. Sci. Math. Educ. 6, 719 (2008).

[10] R. K. Thornton, D. Kuhl, K. Cummings, and J. Marx, Comparing the force and motion conceptual evaluation and the force concept inventory, Phys. Rev. ST Phys. Educ. Res. 5, 010105 (2009).

[11] P. Nieminen, A. Savinainen, and J. Viiri, Force Concept Inventory-based multiple-choice test for investigating students' representational consistency, Phys. Rev. ST Phys. Educ. Res. 6, 020109 (2010); 6, 029903(E) (2010).

[12] C.S. Wallace and J. M. Bailey, Do concept inventories actually measure anything?, Astron. Educ. Rev. 9, 010116 (2010).

[13] P. Heller and D. Huffman, Interpreting the force concept inventory: A reply to Hestenes and Halloun, Phys. Teach. 33, 503 (1995).

[14] D. Huffman and P. Heller, What does the force concept inventory actually measure?, Phys. Teach. 33, 138 (1995).
[15] P. R. Merrifield, Factor analysis in educational research, Rev. Res. Educ. 2, 393 (1974) [http://www.jstor.org/stable/ 1167167].

[16] R. K. Henson, R.M. Capraro, and M. M. Capraro, Reporting practise and use of exploratory factor analysis in educational research journals, in Proceedings of the Annual Meeting of the Mid-South Educational Research Association, Little Rock, Arkansas, 2001, p. 42, www .eric.ed.gov, ID no. ED466780.

[17] J. T. Pohlmann, Use and interpretation of factor analysis in The Journal of Educational Research: 1992-2002, J. Educ. Res. 98, 14 (2004).

[18] R. P. Ang, Development and validation of the TeacherStudent Relationship Inventory using exploratory and confirmatory factor analysis, J. Exp. Educ. 74, 55 (2005).

[19] L. Ding and R. Beichner, Approaches to data analysis of multiple-choice questions, Phys. Rev. ST Phys. Educ. Res. 5, 020103 (2009).

[20] T. A. Schmitt, Current methodological considerations in exploratory and confirmatory factor analysis, J. Psychoeduc. Assess. 29, 304 (2011).

[21] D. Hestenes and I. Halloun, Interpreting the force concept inventory: A response to March 1995 critique by Huffman and Heller, Phys. Teach. 33, 502 (1995).

[22] B. S. Everitt, Multivariate analysis: The need for data, and other problems, Br. J. Psychiatry 126, 237 (1975).

[23] K.D. Kubinger, On artificial results due to using factor analysis for dichotomous variables, Psychology Science 45, 106 (2003) [http://www.pabst-publishers.de/ psychology-science/1-2003/abstract_06.html].

[24] S. M. Kendall, Kendall's Advanced Theory of Statistics (Oxford University Press, New York, 1987).

[25] J.P. Guilford and T.C. Lyons, On determining the reliability and significance of a tetrachoric coefficient of correlation, Psychometrika 7, 243 (1942).

[26] S. P. J. Hayes, Tables of the standard error of tetrachoric correlation coefficient, Psychometrika 8, 193 (1943).

[27] J. Kaltenhauser and Y. Lee, Correlation coefficients for binary data in factor analysis, Geogr. Anal. 8, 305 (1976). 
[28] D. R. Divgi, Calculation of the tetrachoric correlation coefficient, Psychometrika 44, 169 (1979).

[29] B. Muthén and C. Hofacker, Testing the assumptions underlying tetrachoric correlations, Psychometrika 53, 563 (1988).

[30] M. A. Hamdan, The equivalence of tetrachoric and maximum likelihood estimates of $\rho$ in $2 \times 2$ tables, Biometrika 57, 212 (1970).

[31] P. G. N. Digby, Approximating the tetrachoric correlation coefficient, Biometrics 39, 753 (1983).

[32] R. J. Mislevy, Recent developments in the factor analysis of categorical variables, J. Educ. Stat. 11, 3 (1986).
[33] D. G. Bonett and R. M. Price, Inferential methods for the tetrachoric correlation coefficient, J. Educ. Behav. Stat. 30, 213 (2005).

[34] J.L. Horn, A rationale and test for the number of factors in factor analysis, Psychometrika 30, 179 (1965).

[35] J.C. Hayton, D. G. Allen, and V. Scarpello, Factor retention decisions in exploratory factor analysis: A tutorial on parallel analysis, Organ. Res. Meth. 7, 191 (2004).

[36] R.B. Cattell, The scree test for the number of factors, Multivariate Behav. Res. 1, 245 (1966). 